Virginia Commonwealth University

VCU Scholars Compass

2011

\title{
Synthesis of high magnetization FeCo alloys prepared by a modified polyol process
}

Zachary J. Huba

Virginia Commonwealth University

Kyler J. Carroll

Virginia Commonwealth University

Everett E. Carpenter

Virginia Commonwealth University, ecarpenter2@vcu.edu

Follow this and additional works at: http://scholarscompass.vcu.edu/chem_pubs

Cart of the Chemistry Commons

Huba, Z. J., Carroll, K. J., \& Carpenter, E. E. Synthesis of high magnetization FeCo alloys prepared by a modified polyol process. Journal of Applied Physics, 109, 07B514 (2011). Copyright (C) 2011 American Institute of Physics.

\section{Downloaded from}

http://scholarscompass.vcu.edu/chem_pubs/34

This Article is brought to you for free and open access by the Dept. of Chemistry at VCU Scholars Compass. It has been accepted for inclusion in Chemistry Publications by an authorized administrator of VCU Scholars Compass. For more information, please contact libcompass@vcu.edu. 


\title{
Synthesis of high magnetization FeCo alloys prepared by a modified polyol process
}

\author{
Zachary J. Huba, Kyler J. Carroll, and Everett E. Carpenter ${ }^{a)}$ \\ Department of Chemistry, Virginia Commonwealth University, Richmond, Virginia 23284, USA
}

(Presented 15 November 2010; received 24 September 2010; accepted 22 October 2010; published online 24 March 2011)

\begin{abstract}
High magnetization, soft ferromagnetic FeCo alloy nanoparticles were synthesized at various $\mathrm{Fe}$ to Co ratios using a modified polyol process. Transmission electron microscopy images revealed that Fe-rich particles had a cubic shape with a mean particle size of $100 \mathrm{~nm}$, while Co-rich particles had a spherical shape. A maximum saturation magnetization of $212 \mathrm{emu} / \mathrm{g}$ was recorded for both $\mathrm{Fe}_{60} \mathrm{Co}_{40}$ and $\mathrm{Fe}_{75} \mathrm{Co}_{25}$ particles. X-ray diffraction scans at room temperature of synthesized particles were characteristic of body-centered-cubic single-phase FeCo. Variable temperature $\mathrm{x}$-ray diffraction scans under $\mathrm{N}_{2}$ gas revealed an order-disorder transition at $600{ }^{\circ} \mathrm{C}$ and a transition to a face-centered-cubic crystal structure at $1000{ }^{\circ} \mathrm{C}$. (C) 2011 American Institute of Physics. [doi:10.1063/1.3544430]
\end{abstract}

\section{INTRODUCTION}

Soft ferromagnetic materials, with high saturation magnetization are used heavily in today's electronics industry and various engineering applications. ${ }^{1,2}$ Hence, a simple and efficient synthesis method for soft magnetic materials, like iron cobalt $(\mathrm{FeCo})$ nanoparticles, is becoming of great interest. FeCo-based alloys have been prepared several ways in the past, including thermal decomposition, sonochemical reduction, and aqueous reduction by borohydride derivatives. ${ }^{3-5}$ However, the synthesis of single-phase crystals containing metallic iron can be difficult due to iron's tendency to readily oxidize in ambient conditions and form iron oxide. Oxide phase-free $\mathrm{FeCo}$ nanoparticles have been synthesized using the polyol process. ${ }^{6-8}$ Earlier methods for synthesizing FeCo particles utilizing the polyol process used ethylene glycol (EG) as a solvent and a base such as sodium hydroxide $(\mathrm{NaOH})$ under refluxing conditions, and in some cases under inert atmosphere to reduce oxidation. ${ }^{6-8}$

In this work, particles were first synthesized by dissolving the metal precursor salts and then heating the solution under distillation conditions. This resulted in the production of metallic cobalt and in some cases iron oxide nanoparticles. Earlier reports showed that by adding the metal precursors into boiling EG, iron nanoparticles with minimal oxide formation can be synthesized. ${ }^{9}$ Thus in this paper, FeCo particles are synthesized using a similar addition method. When scaled, this method has the ability to produce nanoparticles with numerous potential applications in the electronics and engineering fields.

\section{EXPERIMENTAL PROCEDURES}

In a typical polyol process, metal salts are dissolved in a polyhydric alcohol (polyol) and taken to higher temperatures, usually under an inert atmosphere. ${ }^{10}$ In this work, EG was

\footnotetext{
a) Author to whom correspondence should be addressed. Electronic mail: ecarpenter2@vcu.edu.
}

used as the polyol and $\mathrm{Fe}(\mathrm{II})$ chloride tetrahydrate and cobalt acetate tetrahydrate were the metal precursor salts. Instead of dissolving the metal salts in the polyol first, the metal salts were added into the EG at elevated temperatures under distillation conditions. First $100 \mathrm{~mL}$ of $\mathrm{EG} / \mathrm{NaOH}$ solution was heated under distillation conditions, where all the byproducts were kept in the reaction vessel, for 20 min until a temperature of $195^{\circ} \mathrm{C}$ was reached. At that point, the metal precursor powders were then added into the solution and allowed to react for $20 \mathrm{~s}$. The heat source was then shut off and the solution was then allowed to cool for an hour to about $35^{\circ} \mathrm{C}$. A total metal precursor concentration of 0.035 and $0.070 \mathrm{M}$ was used for each $\mathrm{Fe} / \mathrm{Co}$ ratio. The hydroxide-to-metal molar ratio was varied between 85:1 and 42.5:1. Recovery of synthesized particles was found to be more efficient when done at temperatures slightly elevated over room temperature due to the lower viscosity of the supernatant. To recover the particles, the solution was first centrifuged at $5000 \mathrm{rpm}$ for $4 \mathrm{~min}$. The collected black particles were then washed numerous times with methanol, magnetically separated and allowed to dry at room temperature in a vacuum oven.

Various techniques were used to characterize the synthesized FeCo nanoparticles. To determine size, morphology, chemical composition, and the degree of agglomeration, a Zeiss Libra 120 transmission electron microscope (TEM) with an accelerating voltage of $120 \mathrm{kV}$ coupled with electron energy loss spectroscopy (EELS) was used. The EELS Fe maps were attained at $54 \mathrm{eV}$, and the Co maps at $60 \mathrm{eV}$, which correspond to the expected energy loss of electrons due to inelastic scattering. Size distribution was ascertained using small angle $\mathrm{x}$-ray scattering (SAXS) with a $2 \theta$ scan axis from $-0.5^{\circ}$ to $5^{\circ}$ and processed using EasySAXS software. The SAXS analysis parameters and template were determined through TEM observations. Magnetic property analysis was done using a Lakeshore vibrating sample magnetometer operating at room temperature with a maximum applied field of 10,000 Oe. Thermogravimetric analysis was used to determine the mass of inorganic material for 
magnetization values of synthesized $\mathrm{FeCo}$ particles by annealing samples to $180{ }^{\circ} \mathrm{C}$ at a ramp rate of $10{ }^{\circ} \mathrm{C} / \mathrm{min}$ under $\mathrm{N}_{2}$ gas. Analysis of the crystal phase of synthesized FeCo nanoparticles at room temperature was done using $\theta-2 \theta$ x-ray powder diffraction (XRD) $(\mathrm{Cu} K \alpha$ radiation, $\lambda=1.54506 \AA$ ). For crystal-phase analysis at elevated temperatures an Anton-Paar HTK-1200N high-temperature camera coupled with a TCU-1000N temperature control unit was used under a nitrogen atmosphere. Sample height was adjusted using direct beam bisection at each temperature to account for thermal expansion of the sample and sample holder. For phase identification and phase-percent composition X'Pert Highscore Plus software was used, which utilized the ICDD/JCPDS database. To determine the actual Fe to Co ratio of the product inductively coupled plasma with optical emission spectroscopy (ICP-OES) was used.

\section{RESULTS AND DISCUSSION}

A TEM image of synthesized $\mathrm{Fe}_{75} \mathrm{Co}_{25}$ particles is shown in Fig. 1(A). The particles are cubic in nature and show a mean particle size of $100 \mathrm{~nm}$. In addition to cubic particles, small spherical particles around $20 \mathrm{~nm}$ are also observed. Previous reports using a similar synthetic method identify these to be Fe and iron oxide particles. ${ }^{9}$ The composition of the small spherical particles was confirmed to contain iron through EELS. From the TEM images, a slight degree of particle agglomeration is seen. In contrast to the $\mathrm{Fe}_{75} \mathrm{Co}_{25}$ particles, synthesized $\mathrm{Fe}_{30} \mathrm{Co}_{70}$ particles show a spherical shape, shown in Fig. 1(B). The cubic nature of Ferich particles and the spherical nature of Co-rich particles are consistent with previous reports. ${ }^{6,7} \mathrm{Fe}$ and Co composition maps aquired using EELS were able to identify the synthesized particles as an FeCo alloy. Due to the closeness in expected energy loss, EELS could not be used to determine the percent composition of the FeCo particles. The percent composition of the particles was determined using ICP-OES and is shown in Table I. The final composition was consistent with the ratio of $\mathrm{Fe}$ and Co precursors used. However, the presence of the iron oxide particles seen in the TEM images would suggest that the Fe percent composition in the $\mathrm{FeCo}$ nanoparticles is less than the values reported by ICP-OES.

Average particle size determined using SAXS is $120 \mathrm{~nm}$, which is in good agreement with observed TEM values. Analysis of the SAXS data, calculated a wide-size
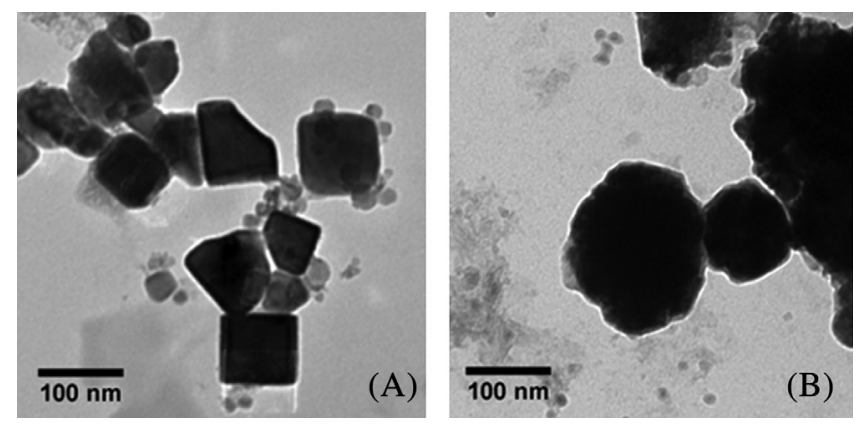

FIG. 1. (A) TEM image of synthesized $\mathrm{Fe}_{75} \mathrm{Co}_{25}$ nanoparticles and (B) TEM image of synthesized $\mathrm{Fe}_{30} \mathrm{Co}_{70}$ nanoparticles.
TABLE I. Initial FeCo ratio and final composition determined by ICP-OES.

\begin{tabular}{ll}
\hline \hline Initial precursor ratio & Final composition \\
\hline $\mathrm{Fe}_{80} \mathrm{Co}_{20}$ & $\mathrm{Fe}_{75} \mathrm{Co}_{25}$ \\
$\mathrm{Fe}_{60} \mathrm{Co}_{40}$ & $\mathrm{Fe}_{60} \mathrm{Co}_{40}$ \\
$\mathrm{Fe}_{50} \mathrm{Co}_{50}$ & $\mathrm{Fe}_{56} \mathrm{Co}_{44}$ \\
$\mathrm{Fe}_{40} \mathrm{Co}_{60}$ & $\mathrm{Fe}_{39} \mathrm{Co}_{61}$ \\
$\mathrm{Fe}_{20} \mathrm{Co}_{80}$ & $\mathrm{Fe}_{30} \mathrm{Co}_{70}$ \\
\hline \hline
\end{tabular}

distribution with a particle-size relative standard deviation of $45.78 \%$. The cause for the wide range of particle size distribution is still unclear. The presence of the small spherical particles in the TEM images [Figs. 1(A) and 1(B)] could be one cause. Also, earlier reports using an injection method observed fast growth rates. ${ }^{9}$ The addition of dry powders used by this synthesis causes nonuniform nucleation and coupled with a fast growth rate could make size control difficult, even for short reaction times, and could be another source of the wide range of particle sizes observed.

A maximum magnetic saturation $\left(M_{s}\right)$ of $212 \mathrm{emu} / \mathrm{g}$ was estimated by plotting $1 / H^{2}$ vs. $M_{s}$ at $\mathrm{FeCo}$ alloys of $\mathrm{Fe}_{60} \mathrm{Co}_{40}$ and $\mathrm{Fe}_{75} \mathrm{Co}_{25}$, which is in agreement with earlier reported $M_{s}$ values and optimal $\mathrm{Fe} / \mathrm{Co}$ ratios. ${ }^{6-8}$ For these particles a coercivity of $\sim 200$ Oe was also recorded. The room-temperature hysteresis loop of $\mathrm{Fe}_{60} \mathrm{Co}_{40}$ nanoparticles is shown in Fig. 2. For alloys below $60 \% \mathrm{Fe}$ content, a decrease in $M_{s}$ was observed.

The peak positions and peak parameters of the room temperature XRD patterns of synthesized FeCo nanoparticles correspond to that of bulk FeCo structure. Variable hightemperature XRD scans were used to determine the changes in crystal phase and crystal structure as a function of temperature (Fig. 3). Up to $200{ }^{\circ} \mathrm{C}$, the dominate crystalline phase is FeCo (PDF 03-065-7519), with only slight peak broadening when compared to the scan at $25{ }^{\circ} \mathrm{C}$, which could be due to thermal motion in the $\mathrm{FeCo}$ lattice. The scan at $400{ }^{\circ} \mathrm{C}$ shows a peak at $35.26^{\circ}$ corresponding to the (311) peak of slightly Fe deficient magnetite $\left(\mathrm{Fe}_{2.957} \mathrm{O}_{4}\right.$, PDF 01-086-1349). The amount of $\mathrm{Fe}_{2.957} \mathrm{O}_{4}$ phase at $400{ }^{\circ} \mathrm{C}$ was calculated to be 24\%. Cobalt oxide (CoO, PDF 01-075-0418) and metallic cobalt (PDF-01-071-4238) peaks were found at $600{ }^{\circ} \mathrm{C}$ along

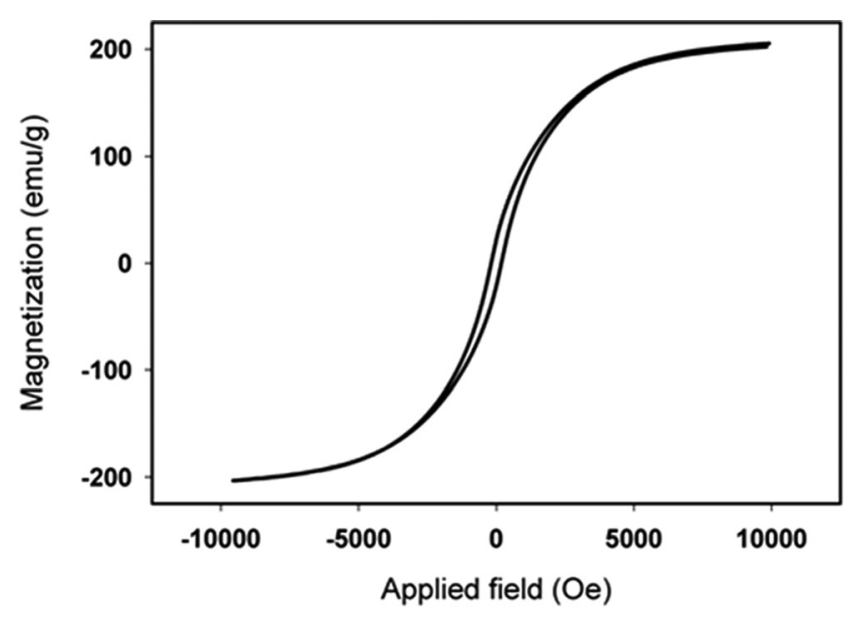

FIG. 2. Hysteresis loop of $\mathrm{Fe}_{60} \mathrm{Co}_{40}$ nanoparticles. 


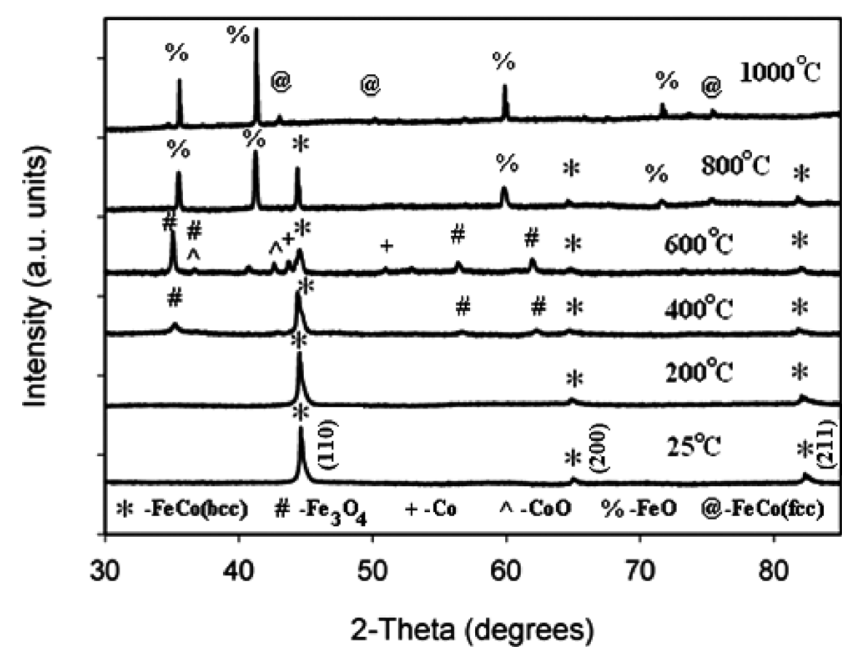

FIG. 3. XRD patterns of FeCo nanoparticles at room and elevated temperatures with $\mathrm{Fe}_{3} \mathrm{O}_{4}, \mathrm{Co}, \mathrm{CoO}$, and $\mathrm{FeO}$ phases identified.

with an increase in the magnetite phase. The percent-phase composition at this temperature was $42 \% \mathrm{Fe}_{2.957} \mathrm{O}_{4}, 34 \%$ $\mathrm{FeCo}, 17 \% \mathrm{CoO}$, and $7 \% \mathrm{Co}$. The (110) peak of FeCo at $44.51^{\circ}$ is observed to have a decrease in intensity and an increase in peak width. The presence of $\mathrm{Co}$ and $\mathrm{CoO}$ peaks at this temperature shows that Co has begun to diffuse out of the FeCo lattice and could be a source of the changes in the FeCo (110) peak seen at $600{ }^{\circ} \mathrm{C}$. This observation corresponds to earlier reports of a low temperature order-disorder transition that occurs around $602{ }^{\circ} \mathrm{C}$. ${ }^{6}$ As the temperature was elevated, the magnetite phase shifted to an $\mathrm{Fe}_{0.911} \mathrm{O}$ (PDF 01-079-2180) phase, with the FeCo peaks becoming narrower and increasing in intensity, at $800{ }^{\circ} \mathrm{C}$. The changes in $\mathrm{FeCo}$ peak width and intensity could signify a betterordered FeCo crystal structure. At $1000{ }^{\circ} \mathrm{C}$, no peaks characteristic of bcc FeCo are present. However, peaks of the $\mathrm{Fe}_{0.911} \mathrm{O}$ phase, and a few low intensity peaks characteristic of Fe (PDF 03-065-4150) with a face-centered-cubic (fcc) crystal structure are present. No profiles for fcc FeCo were found in the database used. After peak fitting, peak positions were shifted to higher angles, and d-spacings were calculated to be lower, than those of bulk fcc Fe. The proposed roomtemperature FeCo crystal structure is bcc Fe with $\mathrm{Fe}$ atoms partly replaced by Co. ${ }^{6}$ This variation in peak and lattice parameters could be explained through the substitution of $\mathrm{Co}$ into the fcc Fe lattice, and could point to fcc FeCo instead of fcc Fe being the phase present at $1000{ }^{\circ} \mathrm{C}$. The phase transition seen at $1000^{\circ} \mathrm{C}$ agrees with earlier reports of a bcc to fcc transition for FeCo particles at $986{ }^{\circ} \mathrm{C} .{ }^{6}$

Since the scans were performed under an inert atmosphere little to no oxide formation might be expected. However, literature from similar synthetic methods suggests that a thin amorphous $4 \mathrm{~nm}$ oxide layer forms on the particles, and this could be a source of some oxide formation seen in the scans at elevated temperatures. Using the reported $4 \mathrm{~nm}$-thick surface oxide layer, 22\% surface oxide content can be calculated for cubic particles with a $100 \mathrm{~nm}$ diameter. This calculated value is significantly lower than the reported $42 \%$ oxide composition for the scan collected at $600{ }^{\circ} \mathrm{C}$. This disparity could be explained by the presence of the small iron oxide particles seen in the TEM images [Fig. 1(A)]. Also washing and storage techniques could add amorphous oxide impurities due to adsorbed oxygen containing species on the particles, as well as an incomplete purging of the heating chamber cannot be ruled out. Even with the presence of oxides, the synthesized FeCo particles still exhibited magnetizations comparable to previous reports, ${ }^{6-8}$ and have potential uses in many commercial and industrial applications.

\section{CONCLUSIONS}

In conclusion, high magnetization FeCo nanoparticles were synthesized using a modified polyol process with the metal precursor salts added to boiling EG. TEM images showed Fe-rich particles with a cubic shape, whereas Corich particles were spherical. TEM and SAXS showed an average particle size of about $100 \mathrm{~nm}$. The presence of smaller spherical Fe or iron oxide particles are seen in TEM images, and identified by EELS. The synthesized nanoparticles exhibited a single-phase FeCo crystal structure at room temperature. XRD scans at elevated temperatures showed an order-disorder transition at $600{ }^{\circ} \mathrm{C}$ and a fcc phase transition at $1000{ }^{\circ} \mathrm{C}$. The formation of iron and cobalt oxide phases was also observed at higher temperatures. A maximum room-temperature $M_{s}$ of $212 \mathrm{emu} / \mathrm{g}$ was achieved in $\mathrm{Fe}_{60} \mathrm{Co}_{40}$ and $\mathrm{Fe}_{75} \mathrm{Co}_{25}$ particles with a coercivity of $200 \mathrm{Oe}$. The high magnetization of the FeCo alloy nanoparticles synthesized using the modified polyol process being reported, make them viable for many applications in the engineering and technology industries.

\section{ACKNOWLEDGMENTS}

This research was supported by the National Science Foundation under Grant No. CHE-0922582. We are also grateful for the help of the VCU Nanomaterials Core Characterization Facility, Dr. Massimo F. Bertino, and Lester W. Brown III.

${ }^{1}$ C. N. Chinnasamy, T. Ogawa, D. Hasegawa, H. T. Yang, S. D. Yoon, V. G. Harris, and M. Takahashi, IEEE Trans. Magn. 43, 3112 (2007).

${ }^{2}$ E. P. Wohlfarth, Ferromagnetic Materials (Elsevier Science, Oxford, 1980), Vol. 2.

${ }^{3}$ G. S. Chaubey, C. Barcena, N. Poudyal, C. B. Rong, J. M. Gao, S. H. Sun, and J. P. Liu, J. Am. Chem. Soc. 129, 7214 (2007).

${ }^{4}$ C. Kuhrt and L. Schultz, J. Appl. Phys. 71, 1896 (1992).

${ }^{5}$ L. Zhang and A. Manthiram, J. Appl. Phys. 80, 4534 (1996).

${ }^{6}$ D. Kodama, K. Shinoda, K. Sato, Y. Konno, R. J. Joseyphus, K. Motomiya, H. Takahashi, T. Matsumoto, Y. Sato, K. Tohji, and B. Jeyadevan, Adv. Mater. 18, 3154 (2006).

${ }^{7}$ D. Kodama, K. Shinoda, K. Sato, Y. Sato, K. Tohji, and B. Jeyadevan, AIP Conf. Proc. 898, 126 (2007).

${ }^{8}$ Q. Nguyen, C. N. Chinnasamy, S. D. Yoon, S. Sivasubramanian, T. Sakai, A. Baraskar, S. Mukerjee, C. Vittoria, and V. G. Harris, J. Appl. Phys. 103, 07D532 (2008).

${ }^{9}$ R. J. Joseyphus, K. Shinoda, D. Kodama, and B. Jeyadevan, Mater. Chem. Phys. 123, 487 (2010).

${ }^{10}$ F. Fievet, J. P. Lagier, B. Blin, B. Beaudoin, and M. Figlarz, Solid State Ionics 32, 198 (1989). 